\title{
Antenatal Diagnosis of Fetal Skeletal Malformation
}

\author{
${ }^{1}$ Ramez Rawhani, ${ }^{2}$ Amal Abdellatif, ${ }^{3}$ Mandy Abushama, ${ }^{4}$ Badreldeen Ahmed
}

\begin{abstract}
Skeletal dysplasia encompasses a heterogamous group of disorders. In this disorder, there are abnormalities involving primarily the fetal skeleton and it may involve other fetal organs. These changes can be lethal or nonlethal. Over 300 types of this abnormality have been described in the literature. The incidence of this condition in the general population is small; however, it contributes significantly to prenatal mortality and morbidity. The etiology and mechanism leading to skeletal dysplasia are not fully understood. Skeletal dysplasia can be inherited as autosomal recessive, dominant, or ever as X-linked disorder and some are of somatic origin. Exposure to external teratogenic factors is a very rare cause of skeletal dysplasia. Antenatal diagnosis of this condition is not easy because of the large number of skeletal dysplasia and the ultrasound finding can overlap between different types of skeletal dysplasia. The newer technology in two-dimensional and three-dimensional ultrasound allows more meticulous studies.

In this article, we will be discussing the most common lethal and nonlethal dysplasia, common methods of evaluating and diagnosing these malformations, and the new technique of noninvasive prenatal diagnosis (NIPD), which can play a major role in prenatal diagnosis of skeletal dysplasia.
\end{abstract}

Keywords: Antenatal ultrasound, Fetal skeletal dysplasia, Prenatal diagnosis.

How to cite this article: Rawhani R, Abdellatif A, Abushama M, Ahmed B. Antenatal Diagnosis of Fetal Skeletal Malformation. Donald School J Ultrasound Obstet Gynecol 2018;12(2):116-123.

Source of support: Nil

Conflict of interest: None

\section{INTRODUCTION}

Skeletal dysplasias are a rare group of disorders associated with malformations and abnormalities in the fetal skeleton. Albeit being rare, skeletal dysplasias have an average prevalence of approximately 5 per 10,000 births, ${ }^{1}$ while having a birth incidence of 1 per $5,000 .{ }^{2}$ Currently, the

\footnotetext{
1,2 Student, ${ }^{3,4}$ Professor

${ }^{1}$ Weill Medical College, Doha, Qatar

${ }^{2}$ Fetal Maternal Center, Doha, Qatar

${ }^{3}$ Weill Medical College, Doha, Qatar; Fetal Maternal Center Doha, Qatar

${ }^{4}$ Weill Medical College, Doha, Qatar; Fetal Maternal Center Doha, Qatar; Qatar University, Doha, Qatar

Corresponding Author: Badreldeen Ahmed, Professor, Weill Medical College, Doha, Qatar; Fetal Maternal Center, Doha Qatar, Qatar University, Doha, Qatar e-mail: profbadreldeen@ hotmail.com
}

number of skeletal dysplasias identified is 436 within 42 groups, with some dysplasias involving genes amounted to be approximately 364 genes to-date. ${ }^{3}$

Over the years, classifications of genetic skeletal disorders revolved around different diagnostic and screening modalities, such as clinical, imaging, and molecular/ genetic modalities, which are still used to this day. A multidisciplinary approach and a specialized unit requiring expertise and involving fetomaternal specialists, radiologists, geneticists, and molecular biologists are vital to tackling diagnosis of fetal skeletal abnormalities. Interpretation of such data is difficult and is rooted in our incomplete understanding of the etiology and mechanism of these skeletal abnormalities; therefore, expertise is crucial. Such units may include surgical specialists in order to address management of the skeletal malformations and their complications.

In this review, we will discuss the most common lethal and nonlethal dysplasia, common methods of evaluating and diagnosing these malformations, and the new technique of NIPD, which can play a major role in prenatal diagnosis of skeletal dysplasia.

\section{DIAGNOSTIC TESTS}

Skeletal dysplasias can be diagnosed prenatally through ultrasonographic evaluation and are thereby confirmed by other means, such as through molecular and genetic testing, or even postnatally through clinical assessment and imaging. Confident diagnosis through ultrasound (US) has become easier with the advancement of the two-dimensional and three-dimensional US, allowing for more meticulous studies. First trimester US is becoming an integral part of routine antennal care in most part of the world. The emphasis on this first trimester scan is on viability, number of fetuses, gestational age, and location of pregnancy. However, nuchal translucency (NT) and other markers of aneuploidy are extremely important components of first trimester scan. First trimester scan is critical in diagnosis of skeletal malformation involving the arms and hands. A condition like Tetra-amelia can be diagnosed during routine first trimester scan. Polydactyly can be seen during first trimester examination. It is associated with trisomy 13 and many other genetic disorders, such as Meckel-Gruber, Ellis-van Creveld, and short rib polydactyly. Table 1 shows a summary of the most common arms and hands defect associated with chromosomal conditions. 
Table 1: A summary of the most common arm and hand defects associated with chromosomal conditions

\begin{tabular}{ll}
\hline Aneuploidy & Anatomical defects in limb \\
\hline Trisomy 21 & $\begin{array}{l}\text { Mostly normal anatomy of the hand } \\
\text { Radial aplasia and overlapping of } \\
\text { Trisomy 18 }\end{array}$ \\
$\begin{array}{l}\text { fingers } \\
\text { Polydactyly }\end{array}$ \\
$\begin{array}{l}\text { Trisomy 13 } \\
\text { Triploidy }\end{array}$ & $\begin{array}{l}\text { Normal anatomy of the hand } \\
\text { Syndactyly }\end{array}$ \\
\hline
\end{tabular}

Additional evaluation is possible by means of molecular analysis, where samples are derived by chorionic villus sampling (CVS) or amniocentesis and should be offered to families and pregnancies where certain risk factors for skeletal dysplasia exist, such as old maternal age, affected/carrier parent, and/or occasionally family history. ${ }^{4}$

Furthermore, fetal magnetic resonance imaging (MRI) is used as an adjunct in special cases, especially due to lack of radiation risk with this method. It is commonly used for a more thorough evaluation of the fetal spine and vertebra, and has some advantages over computed tomography (CT) and US, allowing for a larger and finer scope of view and provides soft tissues contrast and information on fetal lung volume and structure to assess for risk of respiratory insufficiency. ${ }^{5}$ The disadvantages of the fetal MRI are the inability to visualize the fetus in real-time, unlike the US, with the US itself being considered the preferred screening method in the first trimester. ${ }^{5}$ Many might consider the costs of the MRI to be a disadvantage; however, the costs of US and MRI vary greatly in different countries with some countries billing more for US. ${ }^{5}$ Nevertheless, MRI screening prenatally is a relatively new practice, and the medical community is yet to reach consensus on standards and protocols to be followed for its use.

Noninvasive prenatal diagnosis is a recent form of screening and diagnosing skeletal dysplasias and refers to the use of cell free fetal deoxyribonucleic acid (cffDNA) extracted from maternal blood and derived from placental trophoblastic cells that have evaded the fetoplacental barrier and invaded maternal circulation, to detect alleles that are absent in the mother's DNA but present in the baby, either because they have been inherited from the father or arisen de novo. ${ }^{6}$ Applications of NIPD include early fetal sex determination, fetal RhD genotyping in $\mathrm{RhD}$ negative mothers, and screening for inherited diseases and sporadic mutations that give rise to skeletal abnormalities [such as achondroplasia, thanatophoric dysplasia (TD), and Apert syndrome]. ${ }^{7}$ Recent screening capabilities, although more costly, have broadened and allowed for screening and diagnosis of autosomal recessive conditions where parents carry the same mutations and of X-linked conditions. ${ }^{8}$
Noninvasive prenatal diagnosis for achondroplasia and TD using a polymerase chain reaction (PCR) assay devised by the NE Thames Regional Genetics Laboratory was approved by the National Health Service (NHS) in the UK in 2012 and has become a standard test in practice since then. ${ }^{9}$ After the emergence of next-generation sequencing, diagnosis of cases deemed inconclusive by the PCR assay previously and was now made possible with more accuracy and less false-negatives, such as with the addition of a panel that detects all mutations in the fibroblast growth factor-3 (FGFR3) gene (responsible for achondroplasia, TD, hypochondroplasia, and a number of other skeletal abnormalities), the investigation of maternal genomes and cffDNA simultaneously, and the detection of previously undiscovered maternal mosaics. ${ }^{9}$ Noninvasive prenatal diagnosis for next-gen sequencing was approved by the NHS in $2013 .{ }^{10}$ Subsequently, a next-gen panel for the FGFR2 mutation for the detection of Apert syndrome was devised and approved by the NHS. ${ }^{10}$ Since NIPD has been offered for skeletal dysplasia, accuracy of diagnosis has increased and the need for invasive testing for these disorders has been notably reduced. ${ }^{11}$

\section{CLASSIFICATIONS AND LETHALITY OF SKELETAL DYSPLASIA}

The classification of skeletal dysplasia and the understanding of their etiologies has evolved over the decades, especially with the advent of different imaging modalities, and the molecular genetics analyses have been discovered and implemented. With the US being one of the initial screening methods to diagnose skeletal dysplasia prenatally, characterizations of these dysplasias have been made easier by means of measuring and identifying the bones involved and the portions of the limbs affected and evaluating the type of abnormality based on descriptions in Table 2.

One crucial factor that should be determined through US is lethality. This can be achieved by measuring the

Table 2: Terms used to describe the type of bones involved in skeletal dysplasia and their definitions

\begin{tabular}{ll}
\hline Terms & Description \\
\hline Rhizomelia & $\begin{array}{l}\text { Description of the proximal limbs (i.e., humeral, } \\
\text { femoral) when disproportionately shortened in } \\
\text { length }\end{array}$ \\
Mesomelia & $\begin{array}{l}\text { Description of the middle parts of the limbs (i.e., } \\
\text { forearm, leg) when disproportionately shortened }\end{array}$ \\
Acromelia & $\begin{array}{l}\text { Description of the distal limbs (i.e., hands, feet) } \\
\text { when disproportionately shortened }\end{array}$ \\
Micromelia & $\begin{array}{l}\text { A condition in which all segments of the limb are } \\
\text { present but are abnormally shortened }\end{array}$ \\
Campomelia & $\begin{array}{l}\text { A condition in which bowing of the long bones } \\
\text { occurs }\end{array}$ \\
\hline
\end{tabular}


Table 3: Common lethal and nonlethal skeletal dysplasias

\begin{tabular}{|c|c|}
\hline Lethal & Nonlethal \\
\hline $\begin{array}{l}\text { Thanatophoric dysplasia } \\
\text { (most common lethal) }\end{array}$ & $\begin{array}{l}\text { Achondroplasia (most common } \\
\text { nonlethal) }\end{array}$ \\
\hline Achondrogenesis & $\begin{array}{l}\text { Club and rocker-bottom feet } \\
\text { (vertical talus) }\end{array}$ \\
\hline Campomelic dysplasia & Diastrophic dysplasia \\
\hline $\begin{array}{l}\text { Osteogenesis imperfecta } \\
\text { type II }\end{array}$ & $\begin{array}{l}\text { Osteogenesis imperfecta type } \\
\mathrm{I}, \mathrm{III} \text {, and } \mathrm{VI}\end{array}$ \\
\hline Jeune's thoracic dystrophy & Radial ray aplasia/hypoplasia \\
\hline
\end{tabular}

heart to chest circumference (with a circumference $>50 \%$ suggesting lethality) and two ratios: The chest circumference to abdominal circumference ratio, and the femur length to abdominal circumference ratio, with ratios of $<0.7$ and $<0.16$ respectively, being highly suggestive for lethality. ${ }^{12}$ Based on this assessment, parental decisions and management planning can take place. A simple overview of common lethal and nonlethal skeletal dysplasia is listed in Table 3, with a more detailed description on the most commonly encountered dysplasia from both groups.

\section{Lethal Skeletal Dysplasias}

\section{Thanatophoric Dysplasia}

Thanatophoric dysplasia is a lethal, dwarfism skeletal dysplasia characterized by micromelia, characteristic cloverleaf skull deformity (Fig. 1), and narrow thorax. It is an autosomal dominant dysplasia that arises sporadically and has an incidence of approximately 1 in 20,000/50,000 in live births, ${ }^{13}$ making it the most common lethal skeletal dysplasia followed by osteogenesis imperfecta (OI) type II.

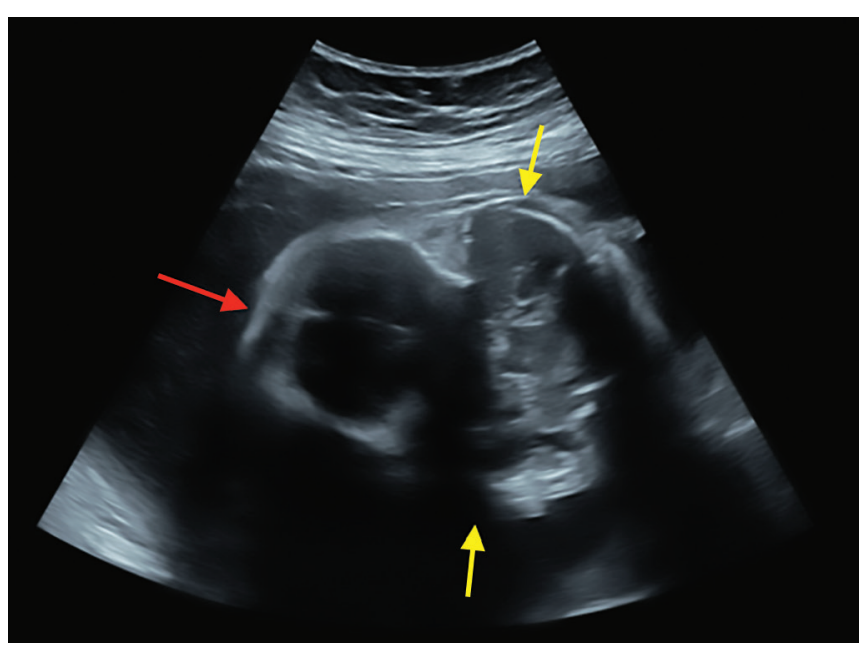

Fig. 1: Thanatophoric dysplasia detected at 27 weeks' gestation with the characteristic three-lobed/cloverleaf skull. The cloverleaf skull is a severe form of craniosynostosis resulting from fusion of all sutures except the metopic suture causing bulging at the topfront of the head (red arrow), and the squamosal sutures causing protrusion from the lower lateral aspect of the skull (yellow arrow)
Thanatophoric dysplasia is subdivided into two types: TD type $\mathrm{I}$ is due to a missense mutation of $742 \mathrm{C} \rightarrow \mathrm{T}$ in the FGFR3 gene, ${ }^{14}$ and is characterized by micromelic (below the 5 th centile at 20 weeks of gestation) ${ }^{15}$ curved femurs, also known as telephone receiver femurs, with or without the finding of cloverleaf skull. Thanatophoric dysplasia type II is due to a missense mutation of $1948 \mathrm{~A} \rightarrow \mathrm{C}$ in the FGFR3 gene, and is characterized by short, straight femurs and a cloverleaf skull. ${ }^{14}$ Other features that can coexist with either type include short ribs, frontal bossing, and bulging abdomen. ${ }^{16}$ Although US diagnosis can be achieved as early as 13 to 15 weeks' gestation, the most confirmed diagnoses occur later on in the second trimester. ${ }^{17}$

The biparietal diameter/femur length (BPD/FL) ratio can be used to screen and to aid in diagnosing skeletal dysplasias, such as TD. Normal BPD/FL ratios decrease to $<3$ during the first trimester, and then continue to decrease to $<2$ as the femur grows, yet in TD the femur growth is stunted from the first trimester leading to a higher-than-expected BPD/FL ratio throughout pregnancy. ${ }^{18}$ Since such ratios can be found in other skeletal dysplasias, further ultrasonographic investigation is needed to support the diagnosis.

Additionally, confirmation of the diagnosis can also be achieved via molecular analysis by means of amniocentesis or CVS, histopathology of bone specimens, and $\mathrm{X}$-ray morphology postdelivery. ${ }^{16}$ Prognosis of TD is very poor with majority of cases being fatal in utero or early on in the neonatal period.

\section{Osteogenesis Imperfecta}

Osteogenesis imperfecta is a skeletal dysplasia that results from a qualitative/quantitative defect in collagen structure and has an overall prevalence of 1 per 10,000 to 20,000 live births. ${ }^{19}$ The phenotypic presentation of the disease is quite variable and dependent on the underlying genetic defect; it ranges from osteopenia, pathologic fractures, and bone deformities leading to demise of the fetus in utero or shortly after birth, especially in OI type II with the involvement of the thorax and causing respiratory insufficiency.

Most of the OI cases arise due to an autosomal dominant mutation in one of the collagen 1 genes: COL1A and COL1A2, while some cases arise due to autosomal recessive mutations in genes that dictate the posttranslational modification and release of type I collagen. ${ }^{19}$ The utility of prenatal diagnosis is most significant when distinguishing between lethal (OI type II) and nonlethal, less severe subtypes (I, III, and IV) for prognosis, and for allowing early, informed, parental decisions.

Osteogenesis imperfecta type II could be screened for by means of prenatal US to detect findings suggestive of bone demineralization and long bone shortening due 
to multiple fractures. Bone demineralization is discernible through the overly-prominent cerebral parenchyma evident below a thin, poorly ossified, cortical calvarial layer. ${ }^{20}$ Multiple rib fractures are commonly seen in OI type II, leading to a narrow, constricted thorax causing pulmonary hypoplasia and contributing significantly to prognosis and lethality, while also producing the characteristic radiographic finding of "beaded ribs" and giving long bones a wrinkly, corrugated appearance. ${ }^{20}$ Conversely, fetuses inflicted with OI type III/IV typically have intact bone ossification and mineralization, and have long bone fractures rather than rib fractures. ${ }^{20}$

\section{Campomelic Dysplasia}

Campomelic dysplasia is a genetic condition involving skeletal and reproductive abnormalities. It has an incidence of approximately 0.5 to 0.9 per 10,000 live births, ${ }^{21}$ and is caused due to a mutation in the SRY-related HMG-box 9 (SOX-9) gene located on chromosome 17 that regulates the activity of other genes involved in the development of the skeleton and the reproductive organs. ${ }^{22}$ The condition is inherited in an autosomal dominant fashion and is lethal in almost all cases.

Diagnosis of campomelic dysplasia can be done as early as 16 weeks' gestational age with ultrasonography. ${ }^{23}$ Ultrasound findings reveal the characteristic finding of campomelia of the long bones, commonly of the femur (Fig. 2), which has given the disorder its name. However, some cases do not have this typical finding and are termed "acampomelic campomelic dysplasia." ${ }^{24}$ Reproductive anomalies are also frequently observed in campomelic dysplasia, with approximately $75 \%$ of the genotypic males displaying ambiguous genitalia and sex reversal. ${ }^{25}$ Other possible findings include a small and narrow thorax with polyhydramnios, 11 pair of ribs, hypoplastic scapulae, flat face with micrognathia, and club feet. ${ }^{26}$
Confirmation of the diagnosis can be accomplished through means of genetic testing by acquiring samples from a CVS or amniocentesis and testing for the SOX-9 gene mutation. ${ }^{26}$ Prognosis is usually poor since majority of cases are lethal mainly due to respiratory compromise. The main causes of this compromise are the deficiency in the tracheobronchial cartilage or due to poor development of the cervical spine. ${ }^{26}$

\section{Nonlethal Skeletal Dysplasias}

\section{Achondroplasia}

Achondroplasia is the most common nonlethal dysplasia defined as rhizomelic shortening of the limbs. The condition has an incidence of 1 per 10,000/30,000 live births, ${ }^{27}$ and is known to be due to a gain of function mutation in the FGFR3 gene, leading to its constant activation where it inhibits the chondrocyte proliferation and leads to endochondral ossification. ${ }^{28}$ While most cases are due to a de novo mutation, inheritance is autosomal dominant and is nonlethal in its heterozygote form, while homozygosity is always lethal owing to the malformation of the thoracic cage leading to eventual respiratory failure.

Diagnosis in utero can be made through US by measuring a short femoral length below third percentile in the second to third trimester as well as the associated US findings. If shortening is noted to be within 18.2 to 26.2 weeks' gestational age, then the case would be considered to be a heterozygotic form, while diagnosis earlier during the second trimester between 14.0 and 16.5 weeks is more commonly associated with homozygotic achondroplasia. ${ }^{29}$ Ultrasound findings in achondroplasia commonly include disproportionate rhizomelic limb shortening (Fig. 3) below the fifth percentile in fetuses, and craniofacial anomalies, such as midface hypoplasia, and macrocephaly with frontal bossing (Fig. 4).
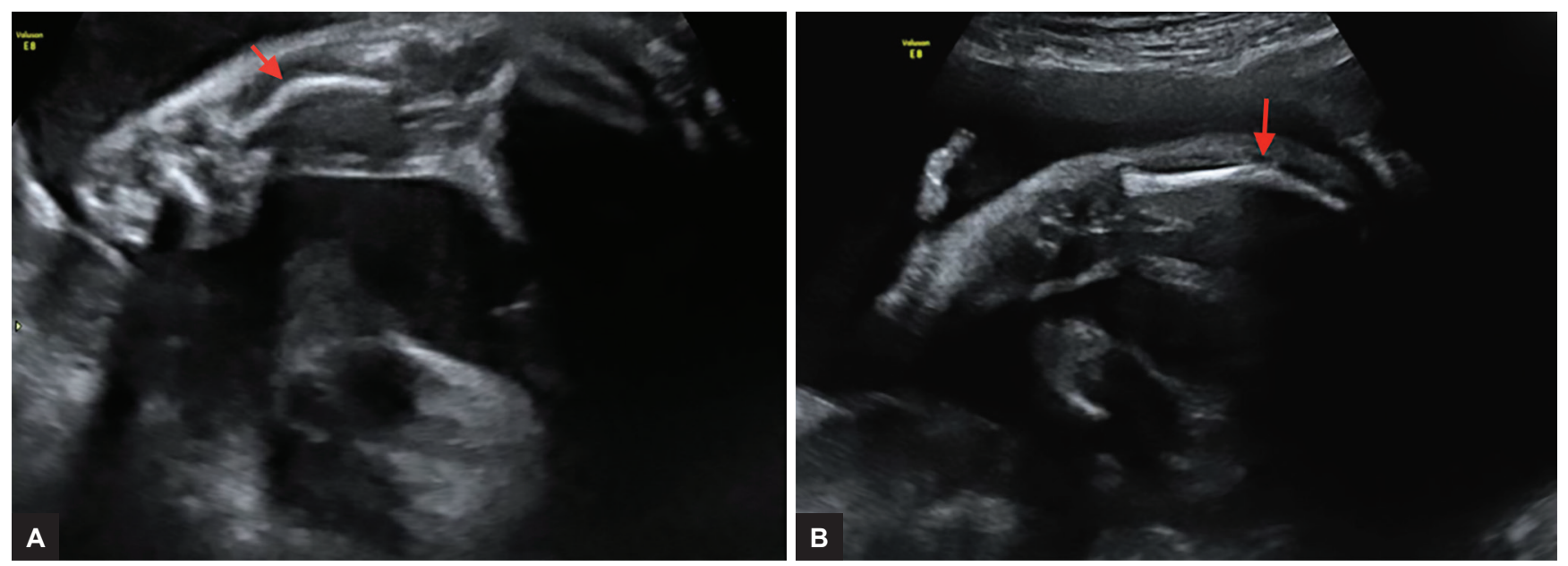

Figs 2A and B: Bowed femur detected at 27 weeks' gestation in a fetus with campomelic dysplasia 


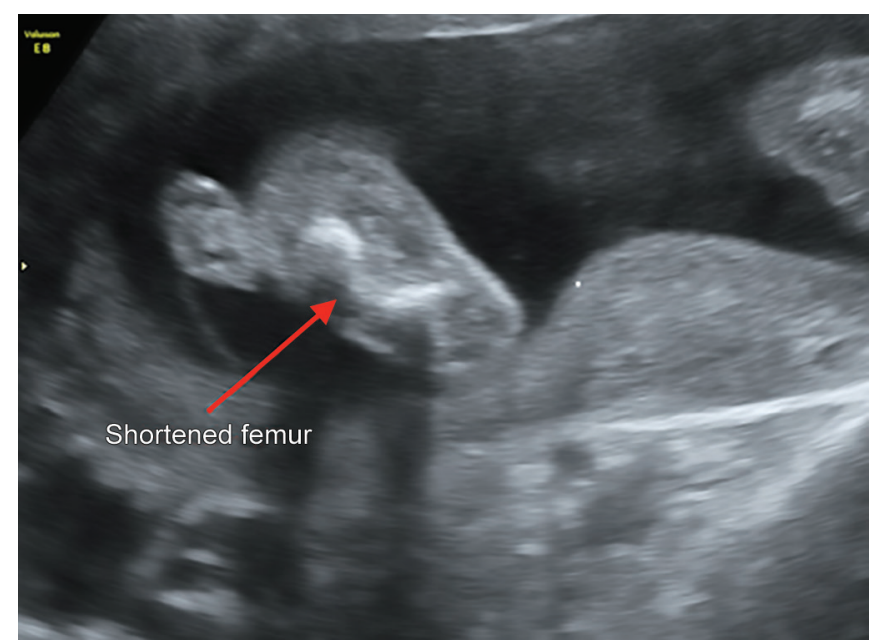

Fig. 3: Achondroplasia detected at 20 weeks' gestation, characterized by rhizomelic limb shortening below the fifth percentile. Polyhydramnios can also be noted

Other findings include depressed nasal bridge, large abdomen, and polyhydramnios that usually develop in the third trimester. If needed, confirmation of diagnosis can be done through genetic/molecular analysis of FGFR3 DNA mutation.

Along with skeletal deformities, individuals with the nonlethal form of achondroplasia also suffer from delayed motor development. However, longevity, intelligence, and cognitive abilities are unaffected in these individuals, allowing for these individuals to have a good prognosis.

\section{Heterogeneous Conditions}

\section{Club Foot}

Talipes or club foot is a foot abnormality involving the bones, musculature, tendons, and tissues in different forms. Its most common form, talipes equinovarus,

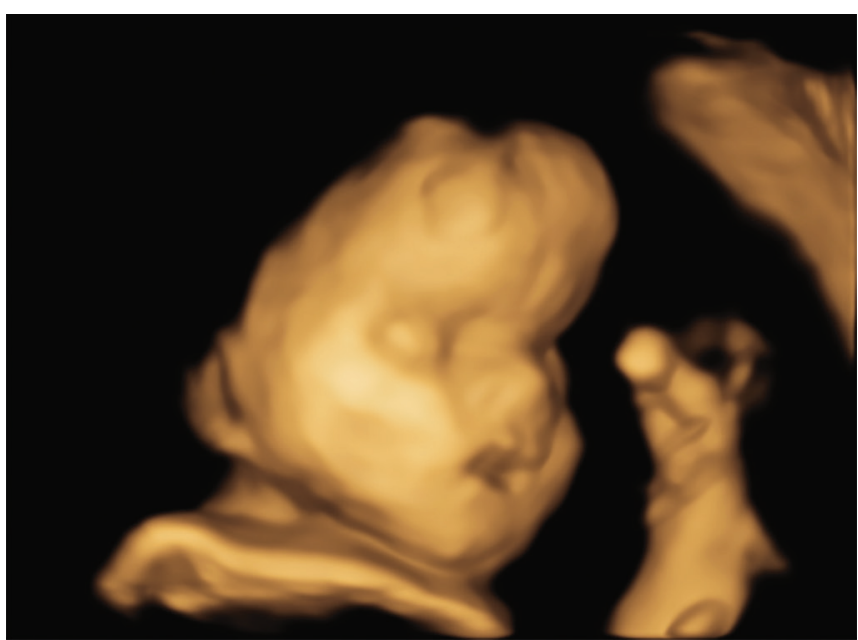

Fig. 4: Fetus with achondroplasia at 27 weeks' gestation with protuberance of the frontal bones of the skull known as frontal bossing

exhibits inversion (medial swinging of the foot) and equinus (plantar flexion) in one or both feet (Fig. 5). Its counterpart, talipes calcaneovalgus, exhibits dorsiflexion of the foot with eversion of the foot. It is considered to be one of the most common congenital orthopedic birth defects affecting on average 3.7 per 1,000 live births. ${ }^{30} \mathrm{Club}$ foot can occur as an isolated finding, although typically associated with chromosomal (such as trisomy 18), neurologic (such as myelomeningocele or spina bifida), skeletal (such as campomelic dysplasia), or syndromic conditions. Spina bifida is particularly common in patients with club foot occurring in approximately 30 to $50 \%$ of cases. ${ }^{31}$ Currently, no single genetic mutation can be attributed to club foot; some hypothesize the involvement of a single gene or genes, while other speculate multifactorial causes involving both environmental and genetics factors.

Diagnosis can be made as early as 12 to 13 weeks' gestational age through transvaginal US by identifying a
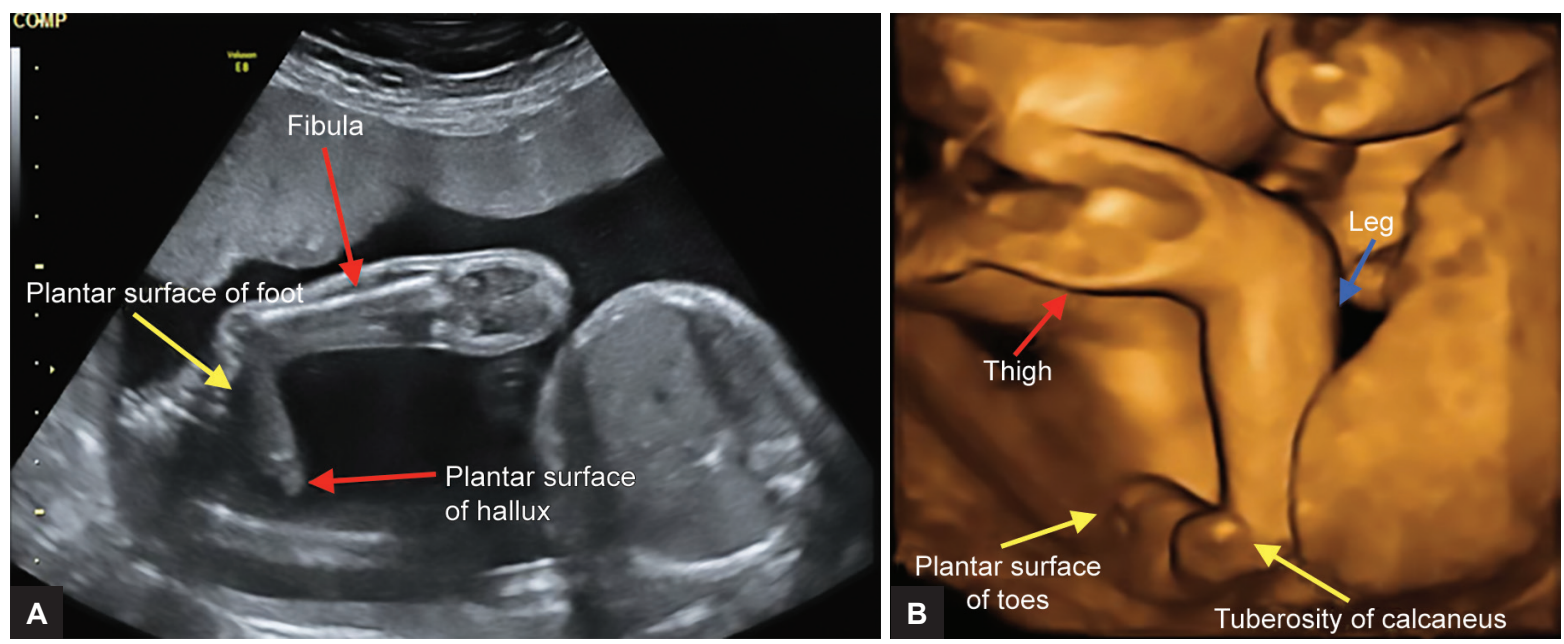

Figs 5A and B: Talipes equinovarus detected in a fetus at 25 weeks' gestation with two-dimensional (left) and threedimensional (right) picture. Inversion of the foot is seen with the plantar and lateral surfaces of the foot in the same plane as the bones of the lower leg 

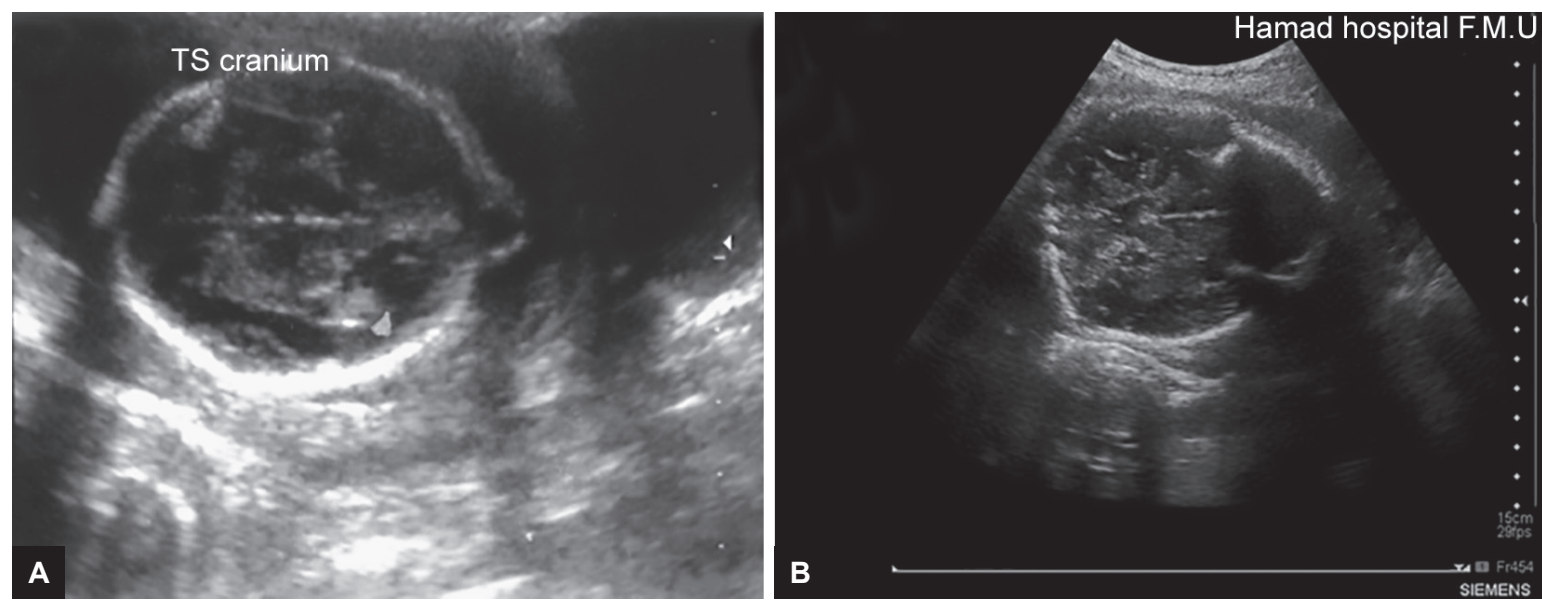

Figs 6A and B: Two-dimensional ultrasound picture of a case of craniosynostosis showing long and narrow head, brachycephaly, frontal bossing, and hypertelorism

deformity in the shape or position of the foot or both feet. This finding should be persistent since some fetus temporarily positions their foot in a similar manner emulating a clubfoot. Since clubfoot occurs in conjunction with other anomalies and conditions, detailed US and fetal echo is recommended.

Prognosis of congenital club foot is dependent on the causes and associations. Club foot itself does not affect longevity, but management is required to better quality of life for these patients. Although the gold standard for the management of club foot is sparing the patient invasive surgery and using the manipulative technique called the Ponseti method, ${ }^{32}$ each case should be assessed by the pediatric orthopedic surgeon to see orthopedic intervention best fits the clinical scenario.

\section{Craniosynostosis}

Craniosynostosis is a heterogamous condition in which there is skull developmental anomaly. The cranial sutures close prematurely, leading to early fusion of the skull plates and subsequent longitudinal expansion of brain parenchyma. The pattern of resultant skull growth pattern, head shape, and direction of cerebral expansion is dependent on the location, number, and timing of suture fusions. For example, in sagittal synostosis, the most common type of craniosynostosis, the skull is longer and narrower posteriorly. ${ }^{33}$

Craniosynostosis has an incidence of 1 per 2000/2500 live births, ${ }^{34}$ occurring as an isolated cranial defect in approximately $85 \%$ of cases and occurs as a part of a syndrome (such as Apert syndrome, Crouzon syndrome, or Pfeiffer syndrome) in about $15 \%$ of cases. ${ }^{35}$ The inheritance of craniosynostosis is dependent on the syndrome that it accompanies, with a majority being autosomal dominant or occurring sporadically. ${ }^{36}$

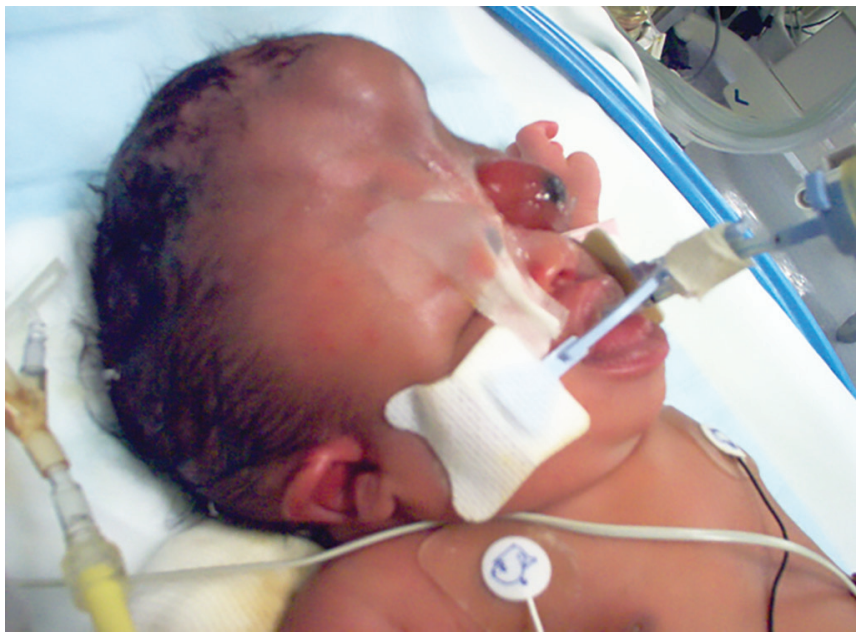

Fig. 7: Newborn patient suffering from craniosynostosis with findings of frontal bossing, exophthalmos, midfacial hypoplasia, and posteriorly displaced ears toward the fused lambdoid sutures

Prenatal diagnosis through US can be achieved as early as 12 weeks' gestation ${ }^{37}$ with characteristic findings depending on the sutures involved. Both twodimensional and three-dimensional US can be used to visualize the cranial sutures and assess for fusion and any other accompanying abnormalities, although the threedimensional US is more superior to two-dimensional US. Common findings during using US examination include long and narrow head, brachycephaly, frontal bossing, midfacial hypoplasia, and hypertelorism (Fig. 6). These fetuses are usually terminated earlier during pregnancy; however, sometimes they present late or termination is not an option for several reasons and the fetus is born at term. Figures 7 and 8 show a case of craniosynostosis at immediate postnatal life.

Further thorough investigation is possible and discernible by MRI, such as temporal depressions and their correlation to fusion of coronal sutures ${ }^{38}$ increased bone density in the forehead region in fetuses with frontal 


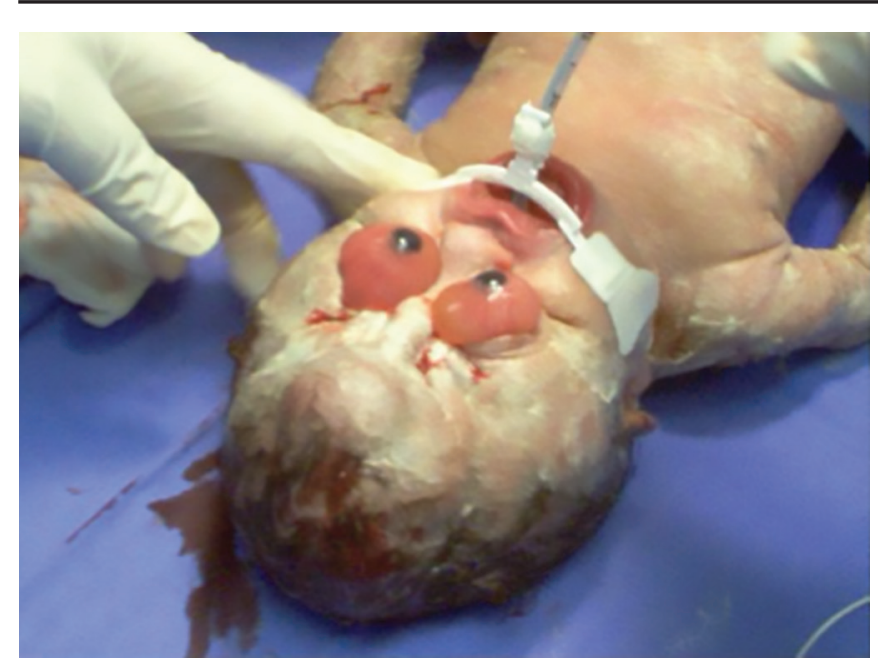

Fig. 8: Newborn patient with craniosynostosis and premature cranial suture closure, exhibiting exophthalmos

(metopic) synostosis, ${ }^{39}$ and better visualization of intracranial changes like ventriculomegaly—a key prognostic factor determining probability of developing hydrocephalus and allowing for management planning ahead of time. ${ }^{38}$ If needed, direct observation of cranial sutures allowing for delineations of calvarial bony structures is also possible through three-dimensional CT scans, and is far more superior than the MRI in this respect. ${ }^{38}$

\section{CONCLUSION}

Skeletal dysplasias are rare disorders, but not infrequent. Diagnosis can be very challenging and is achieved through clinical, molecular/genetic, radiographic, and anthropometric methods. The best approach to diagnosing and managing skeletal abnormalities would be through a specialized multidisciplinary unit. The new era of molecular genetics and NIPD allows patients and physicians to dispense with invasive confirmatory testing, and is of significant utility allowing for genetic diagnosis to be more feasible. This will be of great value in the future and allow for better diagnosis and more precise counseling of the parent.

\section{REFERENCES}

1. Dugoff L, Thieme G, Hobbins JC. Skeletal anomalies. Clin Perinatol 2000 Dec;27(4):979-1005.

2. Krakow D, Rimoin DL. The skeletal dysplasias. Genet Med 2010 Jun;12(6):327-341.

3. Bonafe L, Cormier-Daire V, Hall C, Lachman R, Mortier G, Mundlos S, Nishimura G, Sangiorgi L, Savarirayan R, Sillence D, et al. Nosology and classification of genetic skeletal disorders: 2015 revision. Am J Med Genet 2015 Dec;167(12):2869-2892.

4. Krakow D, Lachman RS, Rimoin DL. Guidelines for the prenatal diagnosis of fetal skeletal dysplasias. Genet Med 2009 Feb;11(2):127-133.

5. Noel AE, Brown RN. Advances in evaluating the fetal skeleton. Int J Women's Health 2014;6:489.
6. Allyse M, Minear MA, Berson E, Sridhar S, Rote M, Hung A, Chandrasekharan S. Non-invasive prenatal testing: a review of international implementation and challenges. Int J Women's Health 2015 Jan;7:113-126.

7. Rafi I, Chitty L. Cell-free fetal DNA and non-invasive prenatal diagnosis. Br J Gen Pract 2009 May;59(562):e146-e148.

8. Verhoef TI, Hill M, Drury S, Mason S, Jenkins L, Morris S, Chitty LS. Non-invasive prenatal diagnosis (NIPD) for single gene disorders: cost analysis of NIPD and invasive testing pathways. Prenat Diagn 2016 Jul;36(7):636-642.

9. Chitty LS, Mason S, Barrett AN, McKay F, Lench N, Daley R, Jenkins LA. Non-invasive prenatal diagnosis of achondroplasia and thanatophoric dysplasia: next-generation sequencing allows for a safer, more accurate, and comprehensive approach. Prenat Diagn 2015 Jul;35(7):656-662.

10. Chitty LS, Lo YD. Noninvasive prenatal screening for genetic diseases using massively parallel sequencing of maternal plasma DNA. Cold Spring Harb Perspect Med 2015 Sep;5(9):a023085.

11. Drury S, Mason S, McKay F, Lo K, Boustred C, Jenkins L, Chitty LS. Implementing non-invasive prenatal diagnosis (NIPD) in a National Health Service Laboratory; from dominant to recessive disorders. Adv Exp Med Biol 2016;924:71-75.

12. Krakow D. Skeletal dysplasias. Clin Perinatol 2015 Jun;42(2):301-319.

13. Orioli IM, Castilla EE, Barbosa-Neto JG. The birth prevalence rates for the skeletal dysplasias. J Med Genet 1986 Aug;23(4):328-332.

14. Chen CP, Chern SR, Shih JC, Wang W, Yeh LF, Chang TY, Tzen CY. Prenatal diagnosis and genetic analysis of type I and type II thanatophoric dysplasia. Prenat Diagn 2001 Feb;21(2):89-95.

15. Chen SW, Chen CP, Wang LK, Chern SR, Wu PC, Chen YN, Lin CJ, Chen WL, Wang W. Perinatal imaging findings and molecular genetic analysis of thanatophoric dysplasia type 1 in a fetus with a c. 2419T> G (p. Ter807Gly)(X807G) mutation in FGFR3. Taiwan J Obstet Gynecol 2017 Feb;56(1):87-92.

16. Bondioni MP, Pazzaglia UE, Izzi C, Di Gaetano G, Laffranchi F, Baldi M, Prefumo F. Comparative X-ray morphometry of prenatal osteogenesis imperfecta type 2 and thanatophoric dysplasia: a contribution to prenatal differential diagnosis. Radiol Med 2017 Nov;122(11):880-891.

17. Schild RL, Hunt GH, Moore J, Davies H, Horwell DH. Antenatal sonographic diagnosis of thanatophoric dysplasia: a report of three cases and a review of the literature with special emphasis on the differential diagnosis. Ultrasound Obstet Gynecol 1996 Jul;8(1):62-67.

18. Wang L, Takai Y, Baba K, Mikami Y, Saito M, Horiuchi I, Konno R, Takagi K, Seki H. Can biparietal diameter-to-femur length ratio be a useful sonographic marker for screening thanatophoric dysplasia since the first trimester? A literature review of case reports and a retrospective study based on 10,293 routine fetal biometry measurements. Taiwan J Obstet Gynecol. 2017 Jun;56(3):374-378.

19. Lim J, Grafe I, Alexander S, Lee B. Genetic causes and mechanisms of osteogenesis imperfecta. Bone 2017 Sep;102:40-49.

20. Milks KS, Hill LM, Hosseinzadeh K. Evaluating skeletal dysplasias on prenatal ultrasound: an emphasis on predicting lethality. Pediatr Radiol 2017 Feb:1-2.

21. Pasupathy M, Radhakrishnan V, Adenwalla HS, Narayanan PV. A case report of acampomelic campomelic dysplasia and operative difficulties in cleft palate reconstruction. Indian J Plast Surg 2016 May;49(2):253. 
22. Hsiao HP, Tsai LP, Chao MC, Tseng HI, Chang YC. Novel SOX9 gene mutation in campomelic dysplasia with autosomal sex reversal. J Formos Med Assoc 2006 Jan;105(12):1013-1016.

23. Schramm T, Gloning KP, Minderer S, Daumer-Haas C, Hörtnagel K, Nerlich A, Tutschek B. Prenatal sonographic diagnosis of skeletal dysplasias. Ultrasound Obstet Gynecol 2009 Aug;34(2):160-170.

24. Moog U, Jansen NJ, Scherer G, Schrander-Stumpel CT. Acampomelic campomelic syndrome. Am J Med Genet A 2001 Dec;104(3):239-245.

25. Mansour S, Hall CM, Pembrey ME, Young ID. A clinical and genetic study of campomelic dysplasia. J Med Genet 1995 Jun;32(6):415-420.

26. Unger S, Scherer G, Superti-Furga A. Campomelic dysplasia. In: Adam MP, Ardinger HH, Pagon RA, Wallace SE, Bean LJH, Stephens K, Amemiya A, editors. SourceGeneReviews ${ }^{\circledR}$ [Internet]. Seattle (WA): University of Washington; 1993-2018.

27. Yang PY, Liao HG, Yeh GP, Hsieh CT. Prenatal diagnosis of achondroplasia with ultrasound, three-dimensional computed tomography and molecular methods. J Med Ultrasound 2012 Sep;20(3):176-179.

28. Sahni M, Ambrosetti DC, Mansukhani A, Gertner R, Levy D, Basilico C. FGF signaling inhibits chondrocyte proliferation and regulates bone development through the STAT-1 pathway. Genes Dev 1999 Jun;13:1361.

29. Patel MD, Filly RA. Homozygous achondroplasia: US distinction between homozygous, heterozygous, and unaffected fetuses in the second trimester. Radiology 1995 Aug;196(2):541-545.
30. Barker S, Chesney D, Miedzybrodzka Z, Maffulli N. Genetics and epidemiology of idiopathic congenital talipes equinovarus. J Pediatr Orthop 2003 Mar;23(2):265-272.

31. Swaroop VT, Dias L. Orthopaedic management of spina bifida-part II: foot and ankle deformities. J Child Orthop 2011 Sep;5(6):403-414.

32. Sanzarello I, Nanni M, Faldini C. The clubfoot over the centuries. J Pediatr Orthop B 2017 Mar;26(2):143-151.

33. Slater BJ, Lenton KA, Kwan MD, Gupta DM, Wan DC, Longaker MT. Cranial sutures: a brief review. Plast Reconstr Surg 2008 Apr;121(4):170e-178e.

34. Greenwood J, Flodman P, Osann K, Boyadjiev SA, Kimonis V. Familial incidence and associated symptoms in a population of individuals with nonsyndromic craniosynostosis. Genet Med 2013 Sep;16(4):302-310.

35. Kim HJ, Roh HG, Lee IW. Craniosynostosis: updates in radiologic diagnosis. J Korean Neurosurg Soc 2016 May;59(3):219-226.

36. Panigrahi I. Craniosynostosis genetics: the mystery unfolds. Indian J Human Genet 2011 May;17(2):48.

37. Delahaye S, Bernard JP, Renier D, Ville Y. Prenatal ultrasound diagnosis of fetal craniosynostosis. Ultrasound Obstet Gynecol 2003 Apr;21(4):347-353.

38. Fjørtoft MI, Sevely A, Boetto S, Kessler S, Sarramon MF, Rolland M. Prenatal diagnosis of craniosynostosis: value of MR imaging. Neuroradiology 2007 Jun;49(6):515-521.

39. Krakow D, Santulli T, Platt LD. Use of three-dimensional ultrasonography in differentiating craniosynostosis from severe fetal molding. J Ultrasound Med 2001 Apr;20(4):427-431. 